\title{
Propionibacterium acnes: Time-to-Positivity in Standard Bacterial Culture From Different Anatomical Sites
}

\author{
Rasha Abdulmassih ${ }^{\mathrm{a}}$, Jina Makadia ${ }^{\mathrm{a}}$, James Como ${ }^{\mathrm{a}}$, Michelle Paulson ${ }^{\mathrm{a}}$, \\ Zaw Min ${ }^{\mathrm{a}}$, Nitin Bhanot ${ }^{\mathrm{a}, \mathrm{b}}$
}

\begin{abstract}
Background: Propionibacterium acnes infections are likely underrecognized and underreported. This is partly because of low clinical suspicion, perceived non-pathogenicity, or lack of adequate culture incubation time. We conducted a study to assess the optimal incubation period to recover $P$. acnes from specimens acquired during the workup of suspected clinical infections.
\end{abstract}

Methods: A 5-year retrospective chart review was conducted between January 2010 and December 2014 at a single tertiary-care hospital. All patient cases from which $P$. acnes was recovered were included for analysis. Source of infection, antibiotic use, and culture time-to-positivity (TTP) were recorded.

Results: Implanted devices comprised the single most common source of $P$. acnes infection. In the majority of cases, $P$. acnes was the only organism identified. The mean incubation TTP for all isolates was 5.73 days.

Conclusions: Standard 5-day culture incubation periods are insufficient to recover $P$. acnes. As a result, $P$. acnes is likely a much more common etiology of a variety of clinical infections than previously reported.

Keywords: Propionibacterium acnes; Time-to-positivity; Prosthetic joint infection; Implanted electronic cardiac device infection

\section{Introduction}

Propionibacterium acnes, an anaerobic, non-sporulating, Gram-positive rod has classically been identified as the etiology of acne vulgaris [1]. In more recent years, however, it has been increasingly recognized as a causative agent of invasive

Manuscript accepted for publication September 27, 2016

aDivision of Infectious Diseases, Allegheny General Hospital, 420 East North Ave., Pittsburgh, PA 15212, USA

${ }^{b}$ Corresponding Author: Nitin Bhanot, Division of Infectious Disease, Allegheny General Hospital, 420 East North Ave., Pittsburgh, PA 15212, USA. Email: Nitin.Bhanot@ahn.org

doi: http://dx.doi.org/10.14740/jocmr2753w infections, including prosthetic joints, cardiac devices and neurosurgically implanted materials [2-4]. P. acnes is a part of the normal human skin microbiota, residing in sebaceous glands and hair follicles, conjunctiva, external ear canal, oral cavity, and gastrointestinal tract $[5,6]$. Similar to other bacterial skin flora such as Staphylococcus species, $P$. acnes has high affinity to adhere to implanted devices by production of biofilm $[7,8]$. This property of $P$. acnes assumes an important role in pathogenesis of infection of prosthetic materials and devices.

Studies have shown that $P$. acnes is responsible for up to $1.9 \%$ of all total shoulder arthroplasty infections [9-11]. In cases of CSF shunt infections, it has been reported to be the third most common pathogen [12]. In cardiac implantable electronic device infections, $P$. acnes has accounted for around $1.2 \%$ of cases [13]. Infections due to $P$. acnes may have been underdiagnosed due to a longer incubation period needed for this organism to grow in standard bacterial culture medium.

We identified a significant number of clinical isolates of $P$. acnes from multiple body sites in our institution; many of them were implicated as pathogens. We sought to compile all cases of probable $P$. acnes infections over a 5-year period at our hospital. Specifically, we wanted to better define the timeto-positivity (TTP) of $P$. acnes in standard cultures, and observe if it differed based on the sites of infection.

\section{Materials and Methods}

A retrospective chart review was conducted at our 600-bed tertiary care institution. All cultures with growth of $P$. acnes isolated over a 5-year period (January 2010 to December 2014) were included for analysis, regardless of the specimen source. The study was conducted as a quality improvement (QI) project after approval from the Institutional Review Board.

Charts associated with each positive $P$. acnes isolate were reviewed, and the following information was collected: patient's gender, diagnosis, antibiotic use, specimen site, and TTP of cultures. These data were tabulated in terms of number of cases of infection per body site, as well as range and median TTP.

\section{Results}

A total of 146 specimens from 121 patients were analyzed. The $146 P$. acnes isolates were categorized, in order of decreasing frequency, into central nervous system, blood, bone and joint, 
Table 1. A Summary of the Anatomical Sites of Propionibacterium acnes Isolates With Respective Time-to-Positivity (TTP)

\begin{tabular}{|c|c|c|c|}
\hline Sites of isolation (total: 146) & $\mathbf{N}(\%)$ & TTP (range in days) & Median (days) \\
\hline Blood & $26(17.8)$ & $4-6$ & 5 \\
\hline \multicolumn{4}{|l|}{ Central nervous system } \\
\hline Epidural tissue & $12(8.2)$ & $3-7$ & 6 \\
\hline Cerebrospinal fluid from ventriculo-peritoneal shunt & $12(8.2)$ & $3-7$ & 5.5 \\
\hline Craniotomy site & $29(19.8)$ & $3-8$ & 6 \\
\hline Brain tissue & $1(0.68)$ & 7 & - \\
\hline Subdural hematoma & $1(0.68)$ & 7 & - \\
\hline \multicolumn{4}{|l|}{ Musculoskeletal } \\
\hline Native joints & $5(3.4)$ & $4-7$ & 5 \\
\hline Prosthetic joints & $11(7.5)$ & $5-6$ & 6 \\
\hline Vertebral spine tissue & $8(5.4)$ & $5-8$ & 7 \\
\hline Skin and soft tissue & $22(15)$ & $3-11$ & 6 \\
\hline Cardiac implantable electronic devices & $8(5.4)$ & $5-10$ & 7 \\
\hline Lymph node & $1(0.68)$ & 6 & - \\
\hline \multicolumn{4}{|l|}{ Abdominal } \\
\hline Biliary drain & $2(1.36)$ & 7 & 7 \\
\hline Peritoneal fluid & $2(1.36)$ & 6 & 6 \\
\hline Arteriovenous graft & $1(0.68)$ & 5 & - \\
\hline Pleural fluid & $5(3.4)$ & $5-8$ & 6 \\
\hline
\end{tabular}

skin and soft tissue, implanted cardiac devices, pleural fluid, abdominal fluid, lymph node, and arteriovenous graft tissues sites of infection (Table 1).

The majority $(122 / 146 ; 83.5 \%)$ of specimens were sampled from sterile sites while the remaining $16 \%(24 / 146)$ were collected from superficial wound swabs. In $85.7 \%$ of all isolates, $P$. acnes was the sole microbe identified. The remainder $(14.3 \%)$ were recovered from superficial wound swabs as a part of polymicrobial culture growth.

The TTP for all $P$. acnes specimens ranged from 3 to 11 days, with a mean of 5.73 days, median of 6 days, and a standard deviation of 1.25 days (Table 1). Male patients comprised $77 \%$ (94) of specimen sources.

Implanted devices accounted for $21.9 \%$ (32/146) of specimen sites, including ventriculoperitoneal shunts $37.5 \%$ (12/32), prosthetic joints 35.4\% (11/32), cardiac implantable electronic devices $25 \%(8 / 32)$, and arteriovenous grafts $3.1 \%$ $(1 / 32)$. All of these patients were treated with antimicrobial agents with activity toward $P$. acnes. All infected cardiac devices were extracted, and patients with prosthetic joint infections underwent explanation of prostheses or debridement with hardware retention. A majority of ventriculoperitoneal shunts were surgically revised or extracted; the infected arteriovenous graft was surgically revised.

\section{Discussion}

Our study affirms that $P$. acnes is commonly associated with prosthetic infections. We additionally found that it may be isolated in many different body sites, including native tissues. In our study, we noted that male gender constituted more than two-thirds of patients with positive $P$. acnes culture. A proposed hypothesis of higher incidence of $P$. acnes infection in males compared to females is more hair follicles and increased sebaceous gland activity secondary to higher androgen levels $[14,15]$.

$P$. acnes frequently requires an incubation period longer than 5 days to grow using routine culture methods, given the slow growth rate of this bacterium [16, 17]. When clinical suspicion is high, extending culture incubation duration beyond the standard 5 days is important, thereby maximizing the recovery of $P$. acnes. It has been previously reported that a minimum of 13 days of culture incubation on both aerobic and anaerobic media is needed to optimally recover $P$. acnes [18]. Therefore, in our hospital when $P$. acnes infection is suspected, we had requested the microbiology laboratory to hold cultures up to 14 days. However, our study suggests that a 6 to10-day incubation period may be sufficient, depending upon the site of infection. Defining an appropriate culture incubation period would be beneficial in decreasing overall laboratory cost and workload while ensuring that clinical infections by $P$. acnes are not overlooked.

Major limitations of this study included its retrospective design and the lack of a uniform extension of the traditional incubation period for all cultures. Because of the relatively small sample size in each infection, the estimated TTP in each culture site cannot be generalized and its interpretation requires 
caution. All $P$. acnes isolates identified beyond 5 days were extended on request by physicians to the microbiology lab. Therefore, we feel that $P$. acnes infections are underestimated. A prospective study implementing universally longer incubation periods is needed to better assess TTP and resultant clinical implications.

\section{Conclusions}

Our data add to the understanding of $P$. acnes as a cause of various infections of both native tissues and prosthetic devices. $P$. acnes TTP is frequently longer than the standard 5-day bacterial culture incubation period; based on our study, this microbe is detectable by routine culture methods within 11 days. Routine implementation of longer incubation periods would likely increase the detection of $P$. acnes and help clinicians choose targeted antimicrobial therapy.

\section{Conflicts of Interest}

There are none to report for all authors.

\section{Grant Support}

None.

\section{References}

1. Kirschbaum JO, Kligman AM. The Pathogenic Role of Corynebacterium Acnes in Acne Vulgaris. Arch Dermatol. $1963 ; 88: 832-833$.

2. Nisbet M, Briggs S, Ellis-Pegler R, Thomas M, Holland D. Propionibacterium acnes: an under-appreciated cause of post-neurosurgical infection. J Antimicrob Chemother. 2007;60(5):1097-1103.

3. Gunthard H, Hany A, Turina M, Wust J. Propionibacterium acnes as a cause of aggressive aortic valve endocarditis and importance of tissue grinding: case report and review. J Clin Microbiol. 1994;32(12):3043-3045.

4. Kanafani ZA, Sexton DJ, Pien BC, Varkey J, Basmania C, Kaye KS. Postoperative joint infections due to Propionibacterium species: a case-control study. Clin Infect Dis. 2009;49(7):1083-1085.

5. Brook I, Frazier EH. Infections caused by Propionibacterium species. Rev Infect Dis. 1991;13(5):819-822.

6. Achermann Y, Goldstein EJ, Coenye T, Shirtliff ME. Pro- pionibacterium acnes: from commensal to opportunistic biofilm-associated implant pathogen. Clin Microbiol Rev. 2014;27(3):419-440.

7. Ramage G, Tunney MM, Patrick S, Gorman SP, Nixon JR. Formation of Propionibacterium acnes biofilms on orthopaedic biomaterials and their susceptibility to antimicrobials. Biomaterials. 2003;24(19):3221-3227.

8. Bayston R, Nuradeen B, Ashraf W, Freeman BJ. Antibiotics for the eradication of Propionibacterium acnes biofilms in surgical infection. J Antimicrob Chemother. 2007;60(6):1298-1301.

9. Levy O, Iyer S, Atoun E, Peter N, Hous N, Cash D, Musa F, et al. Propionibacterium acnes: an underestimated etiology in the pathogenesis of osteoarthritis? J Shoulder Elbow Surg. 2013;22(4):505-511.

10. Singh JA, Sperling JW, Schleck C, Harmsen WS, Cofield RH. Periprosthetic infections after total shoulder arthroplasty: a 33-year perspective. J Shoulder Elbow Surg. 2012;21(11):1534-1541.

11. Wang B, Toye B, Desjardins M, Lapner P, Lee C. A 7-year retrospective review from 2005 to 2011 of Propionibacterium acnes shoulder infections in Ottawa, Ontario, Canada. Diagn Microbiol Infect Dis. 2013;75(2):195-199.

12. Conen A, Walti LN, Merlo A, Fluckiger U, Battegay M, Trampuz A. Characteristics and treatment outcome of cerebrospinal fluid shunt-associated infections in adults: a retrospective analysis over an 11-year period. Clin Infect Dis. 2008;47(1):73-82.

13. Durante-Mangoni E, Mattucci I, Agrusta F, Tripodi MF, Utili R. Current trends in the management of cardiac implantable electronic device (CIED) infections. Intern Emerg Med. 2013;8(6):465-476.

14. Shah NB, Tande AJ, Patel R, Berbari EF. Anaerobic prosthetic joint infection. Anaerobe. 2015;36:1-8.

15. Thiboutot D. Regulation of human sebaceous glands. J Invest Dermatol. 2004;123(1):1-12.

16. Dodson CC, Craig EV, Cordasco FA, Dines DM, Dines JS, Dicarlo E, Brause BD, et al. Propionibacterium acnes infection after shoulder arthroplasty: a diagnostic challenge. J Shoulder Elbow Surg. 2010;19(2):303-307.

17. Lutz MF, Berthelot P, Fresard A, Cazorla C, Carricajo A, Vautrin AC, Fessy MH, et al. Arthroplastic and osteosynthetic infections due to Propionibacterium acnes: a retrospective study of 52 cases, 1995-2002. Eur J Clin Microbiol Infect Dis. 2005;24(11):739-744.

18. Butler-Wu SM, Burns EM, Pottinger PS, Magaret AS, Rakeman JL, Matsen FA, 3rd, Cookson BT. Optimization of periprosthetic culture for diagnosis of Propionibacterium acnes prosthetic joint infection. J Clin Microbiol. 2011;49(7):2490-2495. 